\title{
Influence of hereditary haemochromatosis on left ventricular wall thickness: does iron overload exacerbate cardiac hypertrophy?
}

\author{
K. Rozwadowska' ${ }^{1}$, G. Raczak², K. Sikorska ${ }^{3}$, M. Fijałkowski ${ }^{4}$, \\ D. Kozłowski², L. Daniłowicz-Szymanowicz ${ }^{2}$ \\ ${ }^{1}$ Clinical Centre of Cardiology, University Clinical Centre, Gdansk, Poland \\ ${ }^{2} 2^{\text {nd }}$ Department of Cardiology, Medical University of Gdansk, Poland \\ ${ }^{3}$ Department of Tropical Medicine and Epidemiology, Medical University of Gdansk, Poland \\ ${ }^{4} 1^{\text {st }}$ Department of Cardiology, Medical University of Gdansk, Poland
}

[Received: 26 January 2019; Accepted: 6 February 2019]

Background: The left ventricular (LV) hypertrophy increases the risk of heart failure. Hypertension and infiltrative cardiomyopathies are the well-known reasons of LV hypertrophy. The growing interest of scientists in this issue affects hereditary haemochromatosis $(\mathrm{HH})$, which is characterised by the excess deposition of iron mostly due to HFE gene mutation. The aim of our study was to investigate the possible influence of $H H$ on LV parameters in patients with early-diagnosed (early $\mathrm{HH}$ ) and long-lasting and long-treated (old HH) disease.

Materials and methods: Thirty nine early HH and 19 old HH patients were prospectively enrolled in the study; age- and sex-matched healthy volunteers constituted the appropriate control groups. All participants had echocardiography performed (including three-dimension volume and mass analysis); the iron turnover parameters were measured at the time of enrolment in every $\mathrm{HH}$ patients. Results: Echocardiographic parameters regarding to left atrium (LA), LV thickness, mass and long axis length were significantly higher, whereas $L V$ ejection fraction was lower in early $\mathrm{HH}$ in comparison to healthy persons. In old HH patients the differences were similar to those mentioned before, except LV ejection fraction. The presence of hypertension in both HH groups did not influence echo parameters, as well as diabetes in old $\mathrm{HH}$. The strongest correlation in all HH group was found between the time from $H H$ diagnosis and $L A, L V$ thickness and volumes parameters, but the correlations between iron turnover and echo parameters were non-existent.

Conclusions: Hereditary haemochromatosis, not only long-lasting, but also early-diagnosed, could lead to exacerbation of LV wall thickness and cardiac hypertrophy. This effect is not simply connected with hypertension and diabetes that are frequent additional diseases in these patients, but with the time from HH diagnosis. (Folia Morphol 2019; 78, 4: 746-753)

Key words: hereditary haemochromatosis, echocardiography, left ventricular hypertrophy, heart siderosis, heart morphology, diabetes mellitus, arterial hypertension

Address for correspondence: Dr. L. Daniłowicz-Szymanowicz, $2^{\text {nd }}$ Department of Cardiology, Medical University of Gdansk, ul. Skłodowskiej-Curie 3a, 80-210 Gdańsk, Poland, e-mail: ludwik@gumed.edu.pl 


\section{INTRODUCTION}

The left ventricular (LV) hypertrophy (LVH) is a well-known clinical and morphological parameter that is considered to be the important risk factor for heart failure $[5,8]$. It occurs typically in response to the haemodynamic overload in some physiological and pathological conditions, and hypertension is the most important reason for LVH development [18]. The infiltrative cardiomyopathies are the next well-known reason of LVH. This is a diverse group of cardiac diseases characterised by the deposition of abnormal substances within the heart tissue that causes the ventricular walls to develop either diastolic or, less common, systolic dysfunction. Recently the growing interest of scientists in this issue affects hereditary haemochromatosis $(\mathrm{HH})$, which is characterised by the excess deposition of iron mostly due to HFE gene mutation $[1,12,14]$. Dysfunction of molecules that control iron homeostasis leads to excessive iron absorption in the duodenum and upper section of the small intestine, as well as its maldistribution. As there is no regulatory mechanism for iron excretion from the human body, iron is deposited in a variety of tissues and organs (liver, pancreas, skin, joints, heart) over the course of the disease $[1,11,16]$. Bioactive iron ions produce oxidative stress that destroys involved tissues $[6,13,15]$. Cardiomyocytes, due to intense iron intake, are very susceptible to this type of damage [17]. Symptoms of $\mathrm{HH}$ are nonspecific and typically absent in the early stages, therefore a high degree of clinical suspicion is key to making the diagnosis.

Echocardiography, especially with the use of novel three-dimension (3D) techniques, is one of the most important tools for revealing cardiac abnormalities; $\mathrm{LVH}$ is one of the most noticeable alterations which can be detected by echocardiography and is very common complication of $\mathrm{HH}$. Therefore, the aim of our study was to investigate the possible influence of $\mathrm{HH}$ on LV parameters in different groups of $\mathrm{HH}$ patients: with early-diagnosed disease and with long-lasting and long-treated pathology.

\section{MATERIALS AND METHODS}

\section{Study population}

From November 2014 to November 2018, we prospectively enrolled the patients with newly diagnosed HH (i.e. within 3 months of the initial clinical diagnosis, before receiving $\mathrm{HH}$-specific treatment) [early $\mathrm{HH}$ ] and the patients with $\mathrm{HH}$ treated at least 5 years [o/d $H H$ ]. $\mathrm{HH}$ was initially diagnosed in specialist clinic based on clinical characteristics, abnormal iron turnover parameters and the presence of HFE gene mutations [4]. Healthy age- and sex-matched volunteers constituted two control groups: 26 to early $\mathrm{HH}$ and 24 to old $H H$ patients. All participants underwent classic echocardiography imaging. The protocol of the study was approved by the Local Ethics Committee at the Medical University of Gdansk, and written informed consent was obtained from all participants. The exclusion criteria were as follows: age $<18$ years; history of any cardiological diagnosis (apart from the hypertension). In all HH patients, the levels of iron, serum ferritin, transferrin saturation, haemoglobin, glucose and transaminases level were measured at the time of enrolment.

\section{Echocardiography}

Echocardiography was performed in every person enrolled into the study. The patients were examined in the left lateral decubitus position using a GE VIVID E9 ultrasound system (GE Ultrasound, Horten, Norway) equipped with phased-array transducer (M5S). During the same echocardiographic examination 3D datasets were acquired in apical view using a commercially available matrix-array 3D transducer (4V).

Standard echocardiographic parameters were obtained according to the principles described in recommendations [9]. Data acquisitions were obtained from parasternal long- and short-axis views and the three standard apical views. For each view, three consecutive cardiac cycles were recorded during quiet respiration. Grayscale recordings were optimised for LV evaluation at a frame rate of 50-80 frames/s and only persons with these parameters were included into the further analyses. All echocardiograms were stored digitally and further offline analysis was performed using commercial workstation EchoPAC (v201, GE Healthcare Horten, Norway).

Analysis of 2D parameters. Left atrium (LA) diameters (LADs), indexed LA area (LAA index) and indexed LA volume (LAV index), LV end-diastolic diameters (LVEDD), LV end-systolic diameter (LVESD), intraventricular septal (IVS) and posterior wall (PW) thickness were measured in parasternal view. The relative wall thickness (RWT) was calculated as the sum of anteroseptal and posterior wall thickness divided by the LV end-diastolic dimension. LV diastolic longitudinal length was measured as the distance between the mitral annulus and the apex in end-diastole averaged from the 4-, 2- and 3-chamber apical views. 


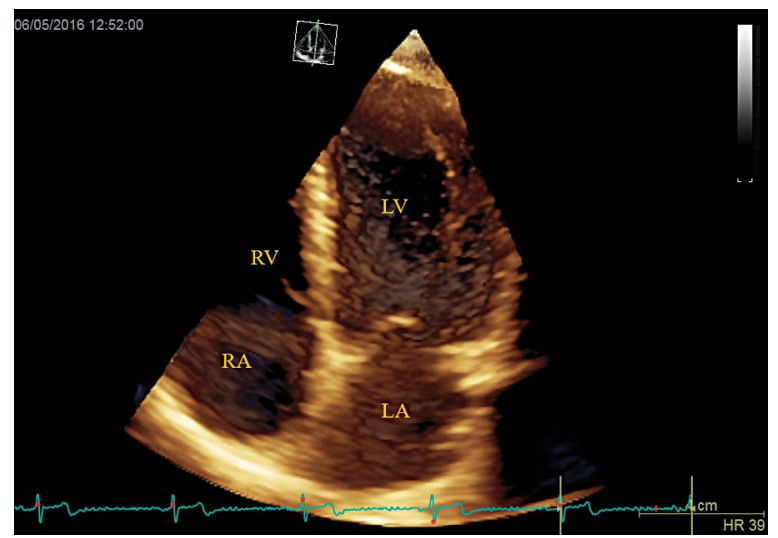

Figure 1. The example of three-dimensional, full-volume echo view for volume, mass and ejection fraction assessment; LA — left atrium; LV — left ventricle; RA — right atrium; RV — right ventricle.

Analysis of 3D parameters. After manual alignment of the three apical views and the short-axis view, points were placed in the mitral annular plane and at the apex, in end-diastole and end-systole. Endocardial and epicardial border were automatically detected by the software, but manually corrected, if necessary. End-diastolic (LVEDV), end-systolic (LVESV) volumes, 3D LV ejection fraction (LVEF) and LV mass (LVM) were measured (Fig. 1).

\section{Statistical analysis}

Continuous data are presented as the median $\left(25^{\text {th }}-75^{\text {th }}\right.$ percentiles), while categorical data are expressed as proportions. We performed the Shapiro-Wilk test to determine whether our data were normally distributed. The majority of the analysed parameters did not have normal data distributions, even after logarithmic data transformation; thus, we selected appropriate statistical analysis methods based on non-parametric tests. Comparisons between groups were performed with the Mann-Whitney $U$ test for continuous variables and Pearson's chi-square test for categorical variables, when appropriate. The correlations between laboratory parameters and echo parameters were evaluated using Spearman's correlation coefficient. P-values less than 0.05 were considered significant. The statistical analysis was conducted with STATISTICA 9.0 (StatSoft, Tulsa OK, USA) and R 2.15.2.

\section{RESULTS}

Thirty nine patients constituted the early $\mathrm{HH}$ group and 19 patients the old $H H$ group, age- and sex-matched healthy volunteers constituted the appropriate control groups (due to the fact that the old $\mathrm{HH}$ patients were older than early $\mathrm{HH}$ patients, there were two respective control groups in our study). The genetic characteristics of the early $\mathrm{HH}$ patients were as follows: 28 patients had the $\mathrm{C} 282 \mathrm{Y} / \mathrm{C} 282 \mathrm{Y}$ genotype, 7 - C282Y/H63D genotype, $3-\mathrm{H} 63 \mathrm{D} / \mathrm{H} 63 \mathrm{D}$ genotype, and $1-\mathrm{C} 282 \mathrm{Y} / \mathrm{wt}$ genotype, whereas the old $\mathrm{HH}$ patients were $\mathrm{C} 282 \mathrm{Y} / \mathrm{C} 282 \mathrm{Y}$, except 2 patients - C282Y/H63D.

Table 1 shows biochemical results of the patients at the time of enrolment. Early $\mathrm{HH}$ had significantly higher ferritin level, whereas the glycaemia level was higher in old $\mathrm{HH}$ group.

In comparison between early $\mathrm{HH}$ group and the healthy persons there were a number of differences regarding to: LA parameters (LADs and LAV index), LV thickness (IVS, PW and RWT) and mass (LVM index), as well as to LV long axis length, which appeared to be significantly higher in early $\mathrm{HH}$ patients. Figures $2 \mathrm{~A}-\mathrm{C}$ demonstrate the example of 2D echo views in early $\mathrm{HH}$ patients, whereas Figures $3 \mathrm{~A}-\mathrm{C}$ - the 2D echogram of the healthy person. 3D echocardiography showed bigger LVESV and lower LVEF in early $\mathrm{HH}$ patients (Table 2). It should be noted that all mentioned echo parameters were within the normal range.

Table 1. HH patients' laboratory characteristics at enrolment

\begin{tabular}{lccc}
\hline & Early $\boldsymbol{H H}(\mathbf{n}=\mathbf{3 9})$ & Old $\mathbf{H H}(\mathbf{n}=\mathbf{1 9})$ & $\mathbf{P}$ \\
\hline Iron $[\mu \mathrm{g} / \mathrm{dL}]$ & $170(148-215)$ & $159(130.5-214)$ & 0.233 \\
Ferritin [ng/mL] & $\mathbf{4 2 1 . 5 ( 2 7 1 - 8 6 0 )}$ & $\mathbf{1 9 2}(\mathbf{8 2 - 2 2 3 )}$ & $\mathbf{0 . 0 0 1}$ \\
TSAT [\%] & $79(60.5-91.8)$ & $71(65.8-81.5)$ & 0.228 \\
Haemoglobin [mg/dL] & $15.1(14-16)$ & $15.1(14.2-16)$ & 0.474 \\
Glycaemia [mg\%] & $\mathbf{9 4}(\mathbf{8 7 - 9 8 )}$ & $\mathbf{1 0 3 ( 9 7 - 1 0 4 )}$ & $<\mathbf{0 . 0 0 9}$ \\
ASPAT [U/L] & $24(18-38)$ & $24(20.5-37)$ & 0.433 \\
ALAT [U/L] & $35(24-64)$ & $26(21-44)$ & 0.209
\end{tabular}

Data are presented as the median $\left(25^{\text {th }}-75^{\text {th }}\right.$ percentile); TSAT — transferrin saturation; ASPAT—aspartate transaminase; ALAT — alanine transaminase 


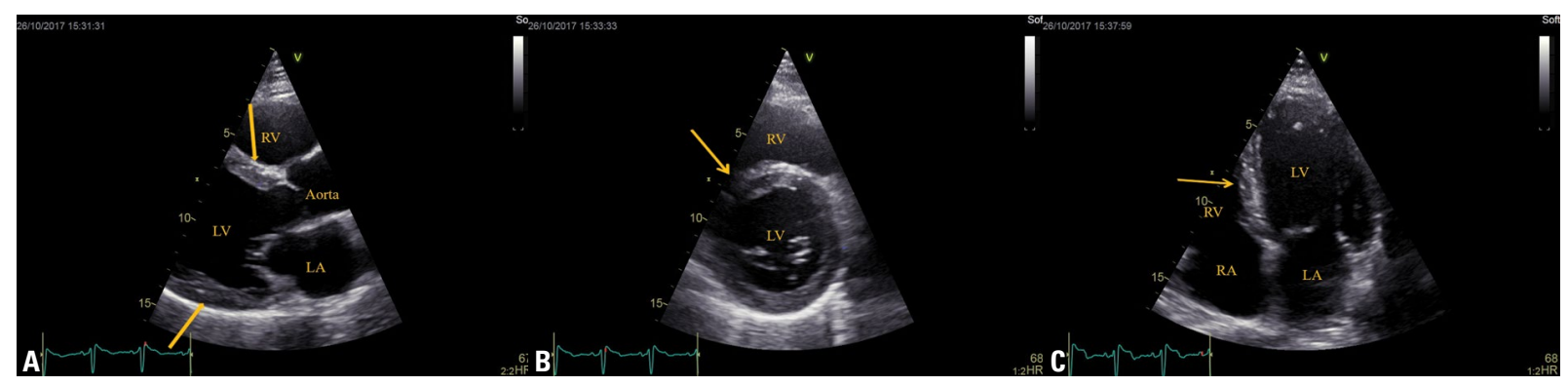

Figure 2. A. The example of parasternal long-axis view in early HH patient (IVS $12 \mathrm{~mm}, \mathrm{PW} 12 \mathrm{~mm}$ ). Arrows point the locations where the measures were taken; B. The example of parasternal short-axis view in early HH patient (IVS $12 \mathrm{~mm}$ ). Arrow points the location where the measure was taken; C. The example apical 4-chamber view in early $\mathrm{HH}$ patient. Arrow points the location where the measure was taken; IVS — intraventricular septum; LA — left atrium; LV — left ventricle; RA — right atrium; RV — right ventricle; PW — posterior wall.

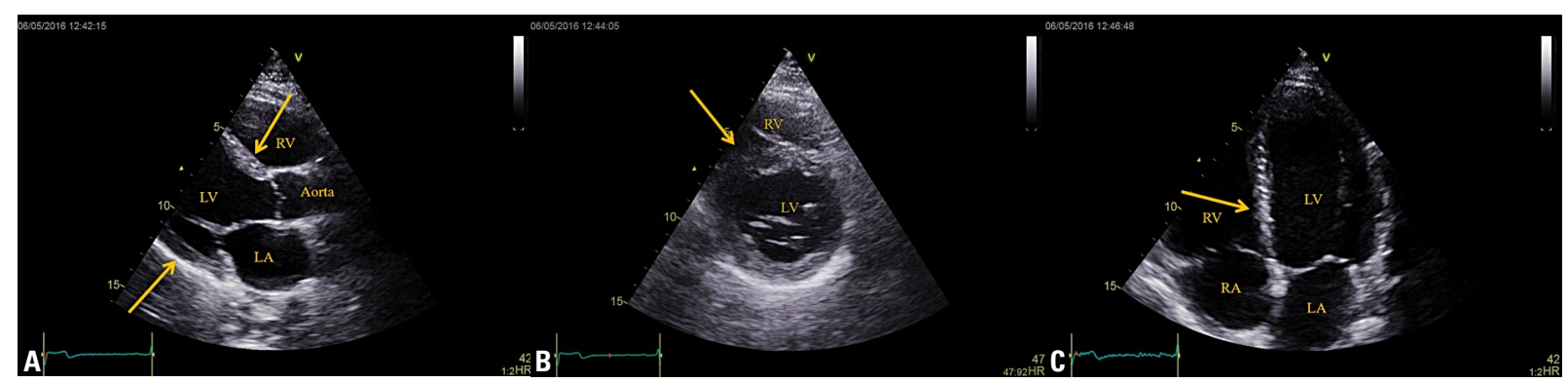

Figure 3. A. The example of parasternal long-axis view in healthy person (IVS $9 \mathrm{~mm}, \mathrm{PW} 9 \mathrm{~mm}$ ). Arrows point the locations where the measures were taken; B. The example of parasternal short-axis view in healthy person (IVS $10 \mathrm{~mm}$ ). Arrow points the location where the measure was taken; C. The example apical 4-chamber view in healthy person. Arrow points the location where the measure was taken; RA — right atrium; RV — right ventricle; LA — left atrium; LV — left ventricle; IVS — intraventricular septum; PW — posterior wall.

Table 2. Comparison of echocardiographic parameters between early $H H$ and healthy groups

\begin{tabular}{|c|c|c|c|}
\hline & Healthy volunteers $(n=26$ ) & Early HH $(\mathrm{n}=39)$ & $\mathbf{P}$ \\
\hline Age [years] & $44(35-53)$ & $38(31-53)$ & 0.199 \\
\hline LADs [mm] & $37(34-39)$ & $42(36-45)$ & $<0.003$ \\
\hline LAA index $\left[\mathrm{cm}^{2} / \mathrm{BSA}\right]$ & $9.0(8.2-9.85)$ & $9.1(7.8-10.1)$ & 0.418 \\
\hline LAV index [mL/BSA] & $21(18-24)$ & $27(23-34)$ & $<0.001$ \\
\hline IVS [mm] & $9(7-9)$ & $10(8-11)$ & $<0.005$ \\
\hline PW [mm] & $8(7-9)$ & $9(7-10)$ & $<0.043$ \\
\hline RWT & $0.37(0.33-0.41)$ & $0.42(0.38-0.46)$ & $<0.001$ \\
\hline LVEDD [mm] & $44(42-47)$ & $45(43-48)$ & 0.111 \\
\hline LVESD [mm] & $28(26-29)$ & $28(25-29)$ & 0.487 \\
\hline LVM index [g/BSA] & $65(54-71)$ & $67(56-92)$ & $<0.045$ \\
\hline LV long axis length [mm] & $74(69-79)$ & 78 (74-81) & $<0.033$ \\
\hline LVEDV [mL] & $105(89-124)$ & $118(97-133)$ & 0.089 \\
\hline LVESV [mL] & $38(33-46)$ & $50(39-56)$ & $<0.005$ \\
\hline LVEF [\%] & $63(61-65)$ & $60(54-61)$ & $<0.001$ \\
\hline
\end{tabular}

Data are presented as the median $\left(25^{\text {th }}-75^{\text {th }}\right.$ percentile); BSA — body surface area; LV — left ventricular; LADs — left atrial diameters; LAA index — left atrium area/BSA; LAV index — left atrium volume/BSA; IVS — intraventricular septum; PW — posterior wall; RWT — relative wall thickness; LVEDD — LV end-diastolic diameter; LVESD — LV end-systolic diameter; LVM index — LV mass/BSA; LVEDV — LV end-diastolic volume; LVESV — LV end-systolic volume; LVEF — LV ejection fraction

In old $H H$ patients the differences were very similar to those mentioned before; there were differences in the LA parameters (LADs, LAA index, LAV index), LV thickness (IVS, PW, RWT, LVM index) and LV long 
Table 3. Comparison of echocardiographic parameters between old $\mathrm{HH}$ and healthy groups

\begin{tabular}{|c|c|c|c|}
\hline & Healthy volunteers ( $n=24$ ) & Old HH (n = 19) & $\mathbf{P}$ \\
\hline Age [years] & $49(43-62)$ & $53(48-65)$ & 0.183 \\
\hline LADs [mm] & $38(34-39)$ & $42(38-43)$ & $<0.004$ \\
\hline LAA index $\left[\mathrm{cm}^{2} / \mathrm{BSA}\right]$ & $9.2(8.0-9.9)$ & $10.5(8.2-12.0)$ & 0.082 \\
\hline LAV index [mL/BSA] & $22(19-28)$ & $35(32-39)$ & $<0.001$ \\
\hline IVS [mm] & $9(7-10)$ & $12(10-12)$ & $<0.001$ \\
\hline PW [mm] & $8(7-10)$ & $11(9-12)$ & $<0.001$ \\
\hline RWT & $0.38(0.34-0.43)$ & $0.44(0.39-0.49)$ & $<0.019$ \\
\hline LVEDD [mm] & $45(42-47)$ & $46(45-49)$ & 0.062 \\
\hline LVESD [mm] & $28(26-30)$ & $29(25-34)$ & 0.215 \\
\hline LVM index [g/BSA] & $68(55-73)$ & 93 (77-102) & $<0.001$ \\
\hline LV long axis length [mm] & $72(66-77)$ & $81(73-82)$ & $<0.004$ \\
\hline LVEDV [mL] & $96(86-120)$ & $93(79-111)$ & 0.225 \\
\hline LVESV [mL] & $36(33-43)$ & $37(28-45)$ & 0.476 \\
\hline LVEF [\%] & $63(61-65)$ & $61(55-65)$ & 0.093 \\
\hline
\end{tabular}

Data are presented as the median $\left(25^{\text {th }}-75^{\text {th }}\right.$ percentile); BSA — body surface area; LV — left ventricular; LADs — left atrial diameters; LAA index — left atrium area/BSA; LAV index — left atrium volume/BSA; IVS — intraventricular septum; PW — posterior wall; RWT — relative wall thickness; LVEDD — LV end-diastolic diameter; LVESD — LV end-systolic diameter; LVM index — LV mass/BSA; LVEDV — LV end-diastolic volume; LVESV — LV end-systolic volume; LVEF — LV ejection fraction

Table 4. Comparison of echocardiographic parameters between $\mathrm{HH}$ patients with and without arterial hypertension

\begin{tabular}{|c|c|c|c|c|c|c|}
\hline & $\begin{array}{c}\text { Early HH without } \\
\text { hypertension }(n=29)\end{array}$ & $\begin{array}{c}\text { Early HH with } \\
\text { hypertension }(n=10)\end{array}$ & $\mathbf{P}$ & $\begin{array}{c}\text { Old } \mathrm{HH} \text { without } \\
\text { hypertension }(\mathrm{n}=7)\end{array}$ & $\begin{array}{c}\text { Old } H H \text { with } \\
\text { hypertension }(n=12)\end{array}$ & $\mathbf{P}$ \\
\hline Age [years] & $33(30-44)$ & $55(53-61)$ & $<0.001$ & $50(42-55)$ & $59(53-65)$ & 0.102 \\
\hline LADs [mm] & 37 (34-42) & $44(42-45)$ & $<0.008$ & $35(34-42)$ & $43(42-45)$ & $<0.018$ \\
\hline LAA index $\left[\mathrm{cm}^{2} / \mathrm{BSA}\right]$ & $8.7(7.8-9.8)$ & $9.6(8.1-10.5)$ & 0.145 & $9.2(8.1-10.7)$ & $11.3(9.1-12.7)$ & 0.123 \\
\hline LAV index [mL/BSA] & $24(22-30)$ & $34(32-38)$ & 0.021 & $32(27-36)$ & $35(34-42)$ & 0.080 \\
\hline IVS [mm] & $10(8-11)$ & $11(8-12)$ & 0.231 & $12(10-13)$ & $12(10-12)$ & 0.481 \\
\hline $\mathrm{PW}$ [mm] & $9(7-10)$ & $10(8-11)$ & 0.151 & $10(9-11)$ & $11(9-12)$ & 0.322 \\
\hline RWT & $0.42(0.38-0.45)$ & $0.42(0.37-0.49)$ & 0.417 & $0.4(0.38-0.45)$ & $0.47(0.4-0.51)$ & 0.170 \\
\hline LVEDD [mm] & 46 (43-48) & $45(42-49)$ & 0.436 & $47(46-50)$ & $45(43-48)$ & 0.146 \\
\hline LVESD [mm] & $28(26-30)$ & $27(25-29)$ & 0.214 & $28(25-32)$ & $29(26-34)$ & 0.428 \\
\hline LVM index [g/BSA] & $66(56-80)$ & $90(56-100)$ & 0.148 & $95(93-103)$ & $84(72-100)$ & 0.149 \\
\hline LV long axis [mm] & 78 (74-83) & $77(74-81)$ & 0.232 & $82(82-85)$ & 77 (72-81) & $<0.046$ \\
\hline LVEDV [mL] & $118(97-133)$ & $123(104-134)$ & 0.458 & $110(97-113)$ & 84 (76-99) & 0.192 \\
\hline LVESV [ml] & $51(39-56)$ & $50(42-56)$ & 0.442 & $39(28-52)$ & $37(30-41)$ & 0.435 \\
\hline LVEF [\%] & $57(54-62)$ & $60(59-60.3)$ & 0.299 & $62(53-67)$ & $60(57-64)$ & 0.414 \\
\hline
\end{tabular}

Data are presented as the median $\left(25^{\text {th }}-75^{\text {th }}\right.$ percentile); BSA — body surface area; LV — left ventricular; LADs — left atrial diameters; LAA index — left atrium area/BSA; LAV index — left atrium volume/BSA; IVS — intraventricular septum; PW — posterior wall; RWT — relative wall thickness; LVEDD — LV end-diastolic diameter; LVESD — LV end-systolic diameter; LVM index — LV mass/BSA; LVEDV — LV end-diastolic volume; LVESV — LV end-systolic volume; LVEF — LV ejection fraction

axis length, but the differences in LVEDV, LVESV and LVEF were not statistically significant (Table 3).

When conducting the separate analysis for early $H H$ and old $H H$ with and without arterial hypertension we did not confirm the differences in LV thickness parameters (IVS, PW, RWT); LVM index was similar in compared patients as well (Table 4).
Among 19 old HH patients, 6 had diabetes. Comparing the echocardiographic parameters between those with and without diabetes, we did not confirm the differences in LV thickness parameters (IVS, PW, RWT); LVM index was similar in compared patients as well (Table 5). Among early $\mathrm{HH}$ patients, only 2 had diabetes; therefore, the statistical analysis was not appropriate for this group. 
Table 5. Comparison of echocardiographic parameters between old $\mathrm{HH}$ patients with and without diabetes mellitus

\begin{tabular}{lccc}
\hline & Old $\mathbf{H H}$ without diabetes $(\mathbf{n}=\mathbf{1 3}$ ) & Old $\mathbf{H}$ with diabetes $\left(\mathbf{n}=\mathbf{6}^{*}\right)$ & $\mathbf{P}$ \\
\hline Age [years] & $\mathbf{5 1}(\mathbf{4 4 - 5 7 )}$ & $\mathbf{6 4 . 5}(\mathbf{5 8 - 6 6 . 5 )}$ & $<\mathbf{0 . 0 3 5}$ \\
LADs [mm] & $\mathbf{4 2}(\mathbf{3 5 - 4 3 )}$ & $\mathbf{4 4}(\mathbf{4 2 - 4 5 )}$ & $\mathbf{0 . 0 3 0}$ \\
LAA index [cm/BSA] & $10.5(8.3-11.5)$ & $11.5(8.6-13.5)$ & 0.192 \\
LAV index [m//BSA] & $35(31-38)$ & $36(34-43)$ & 0.174 \\
IVS [mm] & $11(10-12)$ & $12(11-14)$ & 0.100 \\
PW [mm] & $10(9-11)$ & $12(11-12)$ & 0.090 \\
RWT & $0.42(0.36-0.47)$ & $0.49(0.45-0.52)$ & 0.061 \\
LVEDD [mm] & $47(45-48)$ & $46(42-48)$ & 0.318 \\
LVESD [mm] & $29(25-31)$ & $31(25-34)$ & 0.444 \\
LVM index [g/BSA] & $93(75-96)$ & $95(85-117)$ & 0.227 \\
LV long axis length [mm] & $82(74-82)$ & $79(73-84)$ & 0.462 \\
LVEDV [mL] & $101(88-112)$ & $80(75-83)$ & 0.128 \\
LVESV [mL] & $40(34-49)$ & $29(26-37)$ & 0.167 \\
LVEF [\%] & $60(54-62)$ & $65(58-66)$ & 0.182 \\
\hline
\end{tabular}

Data are presented as the median $\left(25^{\text {th }}-75^{\text {th }}\right.$ percentile); BSA — body surface area; LV — left ventricular; LADs — left atrial diameters; LAA index — left atrium area/BSA; LAV index — left atrium volume/BSA; IVS — intraventricular septum; PW — posterior wall; RWT — relative wall thickness; LVEDD — LV end-diastolic diameter; LVESD — LV end-systolic diameter; LVM index — LV mass/BSA; LVEDV — LV end-diastolic volume; LVESV — LV end-systolic volume; LVEF — LV ejection fraction

Table 6. Correlations between iron turnover and echo parameters in all $\mathrm{HH}$ patients

\begin{tabular}{|c|c|c|c|c|c|c|c|c|}
\hline & \multicolumn{2}{|c|}{ Iron } & \multicolumn{2}{|c|}{ Ferritin } & \multicolumn{2}{|c|}{ Transferrin saturation } & \multicolumn{2}{|c|}{ Time from diagnosis } \\
\hline & $\mathbf{r}$ & $\mathbf{p}$ & $\mathbf{r}$ & $\mathbf{p}$ & $\mathbf{r}$ & $\mathbf{p}$ & $\mathbf{r}$ & $\mathbf{p}$ \\
\hline LADs [mm] & 0.110 & 0.414 & 0.058 & 0.669 & 0.161 & 0.239 & 0.189 & 0.160 \\
\hline LAA index $\left[\mathrm{cm}^{2} / \mathrm{BSA}\right]$ & -0.005 & 0.974 & -0.138 & 0.323 & -0.134 & 0.345 & 0.400 & $<0.003$ \\
\hline LAV index [mL/BSA] & -0.017 & 0.903 & -0.193 & 0.167 & -0.052 & 0.714 & 0.395 & $<0.003$ \\
\hline IVS [mm] & -0.077 & 0.569 & 0.067 & 0.626 & -0.116 & 0.398 & 0.522 & $<0.001$ \\
\hline $\mathrm{PW}[\mathrm{mm}]$ & 0.020 & 0.880 & -0.035 & 0.795 & -0.118 & 0.390 & 0.496 & $<0.001$ \\
\hline RWT & 0.027 & 0.843 & 0.035 & 0.800 & -0.048 & 0.727 & 0.259 & 0.051 \\
\hline LVEDD [mm] & 0.044 & 0.744 & 0.201 & 0.138 & 0.103 & 0.453 & 0.195 & 0.125 \\
\hline LVESD [mm] & -0.036 & 0.790 & -0.081 & 0.553 & -0.044 & 0.752 & 0.211 & 0.115 \\
\hline LVM index [g/BSA] & -0.031 & 0.818 & 0.075 & 0.581 & -0.037 & 0.789 & 0.530 & $<0.001$ \\
\hline LV long axis [mm] & 0.101 & 0.467 & 0.201 & 0.149 & -0.213 & 0.129 & 0.055 & 0.695 \\
\hline LVEDV [mL] & 0.152 & 0.659 & 0.117 & 0.424 & -0.144 & 0.330 & -0.337 & $<0.018$ \\
\hline LVESV [mL] & 0.065 & 0.659 & 0.117 & 0.424 & -0.144 & 0.330 & -0.337 & $<0.018$ \\
\hline LVEF [\%] & 0.068 & 0.640 & 0.008 & 0.957 & -0.041 & 0.780 & 0.219 & 0.130 \\
\hline
\end{tabular}

BSA — body surface area; LV — left ventricular; LADs — left atrial diameters; LAA index — left atrium area/BSA; LAV index — left atrium volume/BSA; IVS — intraventricular septum; PW — posterior wall; RWT — relative wall thickness; LVEDD — LV end-diastolic diameter; LVESD — LV end-systolic diameter; LVM index — LV mass/BSA; LVEDV — LV end-diastolic volume; LVESV — LV end-systolic volume; LVEF — LV ejection fraction

The strongest correlations in all $\mathrm{HH}$ group were found between the time from $\mathrm{HH}$ diagnosis and LA parameters (LADs, LAA index, LAV index), LV thickness parameters (IVS, PW, LVM index) and LV volumes (LVEDV and LVESV), whereas there were no correlations between iron turnover and echo parameters (Table 6).

\section{DISCUSSION}

The most important finding of our study is the observation that in patients with $\mathrm{HH}$ the parameters regarding to LV wall thickness were significantly worse than in age-matched healthy persons. Those differences were independent of hypertension and diabetes, but correlated with the time from initial $\mathrm{HH}$ 
diagnosis. The additional novelty of our study can be found in the demonstration that not only long-lasting $\mathrm{HH}$ leads to $\mathrm{LVH}$, but even early-diagnosed $\mathrm{HH}$ is characterised by higher LV wall thickness parameters in comparison to age- and sex-matched healthy volunteers. It is worth to note, that early HH subgroup of patients was established in short time (within 3 months) after initial diagnosis, before specific $\mathrm{HH}$ treatment. However, despite the lack of any cardiological symptoms and the absence of cardiological history, they presented worse LV thickness parameters. Myocardial iron loading of the heart is well known as a possible complication in late stages of $\mathrm{HH}$ in terms of both diastolic and systolic function [6], but we revealed some differences in heart morphology even in early-diagnosed, not treated $\mathrm{HH}$, where LV thickness parameters were lower than in the old $\mathrm{HH}$ patients, but were prominent and significantly worse than in control group.

When evaluating the overall results of this study, it should be noted, that echocardiographic assessment of $\mathrm{HH}$ patients revealed some other differences in comparison with healthy volunteers. Besides augmentation of rudimentary parameters (IVS, PW, RWT) we detected increased LA parameters, which is known as a typical consequence of $\mathrm{LVH}$ and diastolic dysfunction. In addition, LV long axis length, volume and mass were significantly worse in $\mathrm{HH}$ patients in comparison with controls; these differences have been noted in both $\mathrm{HH}$ groups. All of this undoubtedly suggests that we may deal with haemochromatosis-induced cardiomyopathy rather, than with simple LV thickening. This statement, however, needs to be confirmed in further studies with larger groups of $\mathrm{HH}$ patients and possible follow-up period. According to methodology of our study, it should be underlined, that LV volume and mass were quantified with the use of 3D echocardiography - the technique, which is considered to be comparable with magnetic resonance in relation to abovementioned parameters $[2,7]$.

Finally, it is important to refer to common $\mathrm{HH}$ comorbidities: hypertension and diabetes. LVH is an important marker of hypertension $[10,19,20]$ and diabetes-mediated organ damage [3]. That is why we ruled out the possible impact of these pathologies on LV thickness, demonstrating the lack of significant differences (Tables 4, 5).

We are well aware of potential limitations of our study. This was a small, single-centre study, and therefore its results need to be confirmed in a larger group of patients.

\section{CONCLUSIONS}

Hereditary haemochromatosis, not only long-lasting, but also early-diagnosed, could lead to exacerbation of LV wall thickness and cardiac hypertrophy. This effect is not simply connected with hypertension and diabetes that are frequent additional diseases in these patients, but with the time from $\mathrm{HH}$ diagnosis.

\section{REFERENCES}

1. Andrews NC. Disorders of iron metabolism. N Engl J Med. 1999; 341(26): 1986-1995, doi: 10.1056/ NEJM200004273421716, indexed in Pubmed: 10607817.

2. Bellenger NG, Burgess MI, Ray SG, et al. Comparison of left ventricular ejection fraction and volumes in heart failure by echocardiography, radionuclide ventriculography and cardiovascular magnetic resonance; are they interchangeable? Eur Heart J. 2000; 21(16): 1387-1396, doi: 10.1053/ euhj.2000.2011, indexed in Pubmed: 10952828.

3. Borghetti $G$, von Lewinski $D$, Eaton DM, et al. Diabetic cardiomyopathy: current and future therapies. Beyond glycemic control. Front Physiol. 2018; 9: 1514, doi: 10.3389/ fphys.2018.01514, indexed in Pubmed: 30425649.

4. European Association For The Study Of The Liver. EASL clinical practice guidelines for HFE hemochromatosis. J Hepatol. 2010; 53(1): 3-22, doi: 10.1016/j.jhep.2010.03.001, indexed in Pubmed: 20471131.

5. Gradman AH, Alfayoumi F. From left ventricular hypertrophy to congestive heart failure: management of hypertensive heart disease. Prog Cardiovasc Dis. 2006; 48(5): 326-341, doi: 10.1016/j.pcad.2006.02.001, indexed in Pubmed: 16627048.

6. Gulati V, Harikrishnan P, Palaniswamy C, et al. Cardiac involvement in hemochromatosis. Cardiol Rev. 2014; 22(2): 56-68, doi: 10.1097/CRD.0b013e3182a67805, indexed in Pubmed: 24503941.

7. Hoffmann R, von Bardeleben $S$, ten Cate $F$, et al. Assessment of systolic left ventricular function: a multi-centre comparison of cineventriculography, cardiac magnetic resonance imaging, unenhanced and contrast-enhanced echocardiography. Eur Heart J. 2005; 26(6): 607-616, doi: 10.1093/eurheartj/ehi083, indexed in Pubmed: 15618026.

8. Lloyd-Jones DM, Larson MG, Leip EP, et al. Framingham Heart Study. Lifetime risk for developing congestive heart failure: the Framingham Heart Study. Circulation. 2002; 106(24): 3068-3072, doi: 10.1161/01. cir.0000039105.49749.6f, indexed in Pubmed: 12473553.

9. Mitchell C, Rahko PS, Blauwet LA, et al. Guidelines for Performing a Comprehensive Transthoracic Echocardiographic Examination in Adults: Recommendations from the American Society of Echocardiography. J Am Soc Echocardiogr. 2019; 32(1): 1-64, doi: 10.1016/j. echo.2018.06.004, indexed in Pubmed: 30282592.

10. Nadruz W. Myocardial remodeling in hypertension. J Hum Hypertens. 2015; 29(1): 1-6, doi: 10.1038/jhh.2014.36, indexed in Pubmed: 24804791.

11. Niederau C, Fischer R, Sonnenberg A, et al. Survival and causes of death in cirrhotic and in noncirrhotic 
patients with primary hemochromatosis. N Engl J Med. 1985; 313(20): 1256-1262, doi: 10.1056/NEJM198511143132004, indexed in Pubmed: 4058506.

12. Olsson KS, Norrby A. Comment to: Hepcidin: from discovery to differential diagnosis. Haematologica 2008; 93:90-7. Haematologica. 2008; 93(6): e51; discussion e52, doi: 10.3324/ haematol.12814, indexed in Pubmed: 18515874.

13. Quinlan GJ, Evans TW, Gutteridge JMC. Iron and the redox status of the lungs. Free Radic Biol Med. 2002; 33(10): 1306-1313, doi: 10.1016/s0891-5849(02)00903-6, indexed in Pubmed: 12419462.

14. Rozwadowska K, Daniłowicz-Szymanowicz L, Fijałkowski $\mathrm{M}$, et al. Can two-dimensional speckle tracking echocardiography be useful for left ventricular assessment in the early stages of hereditary haemochromatosis? Echocardiography. 2018; 35(11): 1772-1781, doi: 10.1111/ echo.14141, indexed in Pubmed: 30255609.

15. Stickel F, Osterreicher $\mathrm{CH}$, Datz C, et al. Prediction of progression to cirrhosis by a glutathione S-transferase P1 polymorphism in subjects with hereditary hemochromatosis. Arch Intern Med. 2005; 165(16): 1835-1840, doi: 10.1001/ archinte.165.16.1835, indexed in Pubmed: 16157826.
16. Strohmeyer G, Niederau C, Stremmel W. Survival and causes of death in hemochromatosis. Observations in 163 patients. Ann N Y Acad Sci. 1988; 526: 245-257, doi: 10.1111/j.1749-6632.1988.tb55510.x, indexed in Pubmed: 3389643.

17. Tsushima RG, Wickenden AD, Bouchard RA, et al. Modulation of iron uptake in heart by L-type Ca2 + channel modifiers: possible implications in iron overload. Circ Res. 1999; 84(11): 1302-1309, doi: 10.1161/01.res.84.11.1302, indexed in Pubmed: 10364568.

18. Vasan R. The role of hypertension in the pathogenesis of heart failure. Arch Intern Med. 1996; 156(16): 1789, doi: 10.1001/archinte.1996.00440150033003.

19. Weber T, Protogerou A. Left ventricular hypertrophy, arterial stiffness and blood pressure: exploring the Bermuda Triangle. J Hypertens. 2019; 37(2): 280-281, doi: 10.1097/ HJH.0000000000001973, indexed in Pubmed: 30640868.

20. Williams B, Mancia G, Spiering W, et al. ESC Scientific Document Group . 2018 ESC/ESH Guidelines for the management of arterial hypertension. Eur Heart J. 2018; 39(33): 3021-3104, doi: 10.1093/eurheartj/ehy339, indexed in Pubmed: 30165516. 